\title{
Involvement of the ipsilateral and contralateral cerebellum in the acquisition of unilateral classical eyeblink conditioning in guinea pigs
}

\author{
Bo HU' ${ }^{1,2}$, Xi LIN ${ }^{2}$, Lü-shuai HUANG ${ }^{2}$ Li YANG ${ }^{2}$, Hua FENG ${ }^{1}$, Jian-feng SUI ${ }^{2, *}$ \\ ${ }^{1}$ Department of Neurosurgery, Southwest Hospital, Third Military Medical University, Chongqing 400038, China; ${ }^{2}$ Department of Physiology, College of \\ Basic Medicine, Third Military Medical University, Chongqing 400038, China
}

\begin{abstract}
Aim: The aim of this study was to evaluate the relative contributions of the ipsilateral and contralateral cerebellum to the acquisition of unilateral classical eyeblink conditioning (EBCC).

Methods: The unilateral EBCC was achieved using a binaural tone conditioned stimulus (CS) paired with a left airpuff unconditioned stimulus (US). A high-resolution potentiometer was used to monitor eyeblink responses. Guinea pigs received one CS-US session followed by three CS-US sessions (sessions 2 to 4), during which microinjections of muscimol, a $\mathrm{GABA}_{\mathrm{A}}$ receptor agonist, were performed to reversibly inactivate the cerebellum unilaterally prior to training. To test whether any learning had occurred during these inactivation sessions, training was continued for six more CS-US sessions (sessions 5 to 10) without any inactivation.

Results: Animals with inactivation of the left cerebellum had no signs of left conditioned response (CR) during sessions 2 to 4, and their CR acquisition during sessions 5 to 10 was not distinguishable from that of control animals during sessions 2 to 7. In contrast, animals with inactivation of the right cerebellum acquired left CRs during sessions 2 to 4 , although their CR acquisition was significantly retarded during session 2. In addition, microinjections of muscimol into the right cerebellum did not affect left neuro-behavioral activity. Finally, microinjections of muscimol into either the left or the right cerebellum did not affect the performance of tone-airpuff evoked unconditioned response (UR).

Conclusion: In contrast to the essential role of the ipsilateral cerebellum, the contralateral cerebellum is potentially involved in the acquisition of unilateral EBCC during the early stage of training.
\end{abstract}

Keywords: eyeblink; classical conditioning; acquisition; muscimol; deep cerebellar nuclei; cerebellar cortex Acta Pharmacologica Sinica (2009) 30: 141-152; doi: 10.1038/aps.2008.18; published online 5th January 2009

\section{Introduction}

Classical eyeblink conditioning (EBCC) in rodents is an excellent model system for investigating the neural substrates of motor learning. EBCC involves paired presentations of a conditioned stimulus (CS) with an aversive unconditioned stimulus (US). Numerous lines of evidence have consistently implied that the cerebellum is the essential brain region for the acquisition of EBCC. Convergence of the CS and the US signals at the cerebellar cortex and deep cerebellar nuclei $(\mathrm{DCN})$ induces plasticity in these regions and results in the emergence of a conditioned eyeblink response (CR), which

\footnotetext{
* Correspondence to Prof Jian-feng SUI.

E-mail jfsui@163.com

Received 2008-09-22 Accepted 2008-11-27
}

is similar to the US evoked unconditioned response (UR $)^{[1]}$.

Because of the double decussation of most cerebellar input and output signals, the prevailing hypothesis is that the plasticity involved in CR emergence occurs in the cerebellum ipsilateral to the applied side of the US during the acquisition of unilateral EBCC. This hypothesis has been examined repeatedly via different approaches, including single-unit recording $^{[2,3]}$, lesions ${ }^{[4,5]}$, and inactivation ${ }^{[6-9]}$.

However, this hypothesis has been challenged by the suggestion that plasticity occurs not only in the unilateral cerebellum ipsilateral to but also in the unilateral cerebellum contralateral to the applied side of the US during the acquisition of unilateral EBCC. It had been reported that lesions made in the unilateral cerebellum of rabbits abolished ipsilateral CRs, whereas learning was facilitated when training was switched to the contralateral eye ${ }^{[10]}$. Results from a recent 
functional imaging study of rabbits revealed activation in both the ipsilateral and the contralateral cerebellum during the acquisition of unilateral EBCC ${ }^{[11]}$. Moreover, increased expression of learning-related genes was observed in both sides of the cerebellum in mice ${ }^{[12]}$, which further supports the notion that some degree of plasticity occurs in the cerebellum contralateral to the applied side of the US. Several positron-emission tomography (PET) studies, as well as a single functional magnetic resonance imaging (fMRI) study in humans, have also shown significant changes in bilateral cerebellar activity during the acquisition of unilateral EBCC $^{[13-15]}$, indicating that plasticity occurring in the contralateral cerebellum might exist in various mammalian species. However, at this time, it remains unclear (1) whether the plasticity occurring in the contralateral cerebellum is involved in the acquisition of unilateral EBCC and, if so, (2) what importance contralateral involvement has relative to ipsilateral involvement.

Muscimol (MSC) is well known to temporarily inhibit the activity of neurons that express $\mathrm{GABA}_{\mathrm{A}}$ receptors by hyperpolarizing somata and dendrites by increasing $\mathrm{Cl}^{-}$ conductance ${ }^{[16]}$. In the cerebellar cortex, granule cells are by far the most numerous cells that express $\mathrm{GABA}_{\mathrm{A}}$ receptors ${ }^{[17]}$. Additionally, most synaptic inputs to the DCN are also mediated by $\mathrm{GABA}_{\mathrm{A}}$ receptors ${ }^{[18]}$. Consequently, in the current study, we used local microinjections of muscimol to reversibly inactivate the unilateral cerebellum of albino guinea pigs during the acquisition of unilateral EBCC. The relative contributions of the ipsilateral and contralateral cerebellum to the acquisition of unilateral EBCC were then evaluated. Results of the current study will provide further insight into our understanding of the neural substrates of unilateral EBCC.

\section{Materials and methods}

\section{Subjects}

Thirty male Dunkin-Hartley albino guinea pigs weighing 500-550 $\mathrm{g}$ at the time of surgery were used as subjects. Before the experiment and between conditioning sessions, the guinea pigs were individually housed in standard plastic cages in a colony room with a 12:12 light/dark cycle. Water and food were available. All experiments were conducted between 8:00 AM and 6:00 PM during the lights-on phase and were carried out in a blind fashion. All experimental procedures were approved by the Animal Care Committee of the Third Military Medical University and were in accordance with the principles outlined in the NIH Guide for the Care and Use of Laboratory Animals.

\section{Surgical procedures}

Surgical procedures were performed in an aseptic environment using sterilized surgical tools. Guinea pigs were anesthetized with a mixture of ketamine $(80 \mathrm{mg} / \mathrm{kg}$, ip) and xylazine $(5 \mathrm{mg} / \mathrm{kg}$, ip) and their heads were secured in a stereotaxic apparatus (SR-6N, Narishige, Tokyo, Japan). A longitudinal incision then was made to reveal the skull. According to an atlas of the guinea pig brain ${ }^{[19]}$, four stainless steel skull anchoring screws were implanted together with two stainless steel guiding cannulae aimed bilaterally approximately $1.0 \mathrm{~mm}$ dorsal to the $\mathrm{DCN}$ [stereotaxic coordinates: $\mathrm{AP}=-3.0 \mathrm{~mm}$ from frontal zero plane (APO), $\mathrm{ML}= \pm 2.5 \mathrm{~mm}$ from midline; $\mathrm{DV}=-5.5 \mathrm{~mm}$ from skull surface]. A Plexiglas headstage $(1.0 \mathrm{~cm} \times 1.0 \mathrm{~cm} \times 0.5 \mathrm{~cm}$, designed to secure the animal's head and to hold the airpuff pipe) was attached to the anchoring screws using dental cement. A pair of removable stainless steel rods was inserted into the guiding cannulae to prevent them from clogging. Finally, a small nylon loop was sutured into, but not through, the edge of the left upper eyelid. After the surgery, guinea pigs were allowed one week of recovery and were observed and weighed daily during this period. In the current study, all guinea pigs gained weight and showed no abnormal behavior.

\section{Behavioral procedures}

As suggested by some previous studies, initial training for one eye prior to ipsilateral cerebellar lesion is necessary when evaluating the contralateral cerebellar contribution to the acquisition of unilateral $\mathrm{EBCC}^{[4,10,20]}$. Consequently, in this study our behavioral procedures consisted of three stages of training (Figure 3).

After one week of postoperative recovery, guinea pigs were adapted to the experimental environment for 2 days, 90 $\mathrm{min} /$ day. After these adaptation days, the animals received one daily tone-airpuff conditioning session (session 1). The animals were then divided into three groups in a blind fashion.

The first group ( $n=10$; termed the 'L-MSC' group) received three daily tone-airpuff conditioning sessions (sessions 2 to 4 ), during which the left cerebellum was reversibly inactivated with microinjections of muscimol (see Drug microinjections). These guinea pigs then received three days of rest, followed by six more daily conditioning sessions (sessions 5 to 10) without any microinjection to test whether learning had occurred during the previous three inactivation sessions. Finally, the guinea pigs received a last conditioning session (session 11) in which muscimol was again microinjected into the left cerebellum to test the effects of left cerebellar inactivations on retention of the established left CRs. 
The second group ( $n=10$; termed the 'R-MSC' group) also received three daily tone-airpuff conditioning sessions (sessions 2 to 4 ), during which the right cerebellum was reversibly inactivated with microinjections of muscimol (see Drug microinjections). These guinea pigs then received three days of rest, followed by six more daily conditioning sessions (sessions 5 to 10) without microinjections to test whether learning had occurred during the previous three sessions with inactivations. In the end, these guinea pigs received a last conditioning session (session 11) in which muscimol was microinjected into the right cerebellum to test the effects of right cerebellar inactivations on retention of the established left CRs.

The third group ( $n=10$; termed the 'Control' group) received three daily tone-airpuff conditioning sessions (sessions 2 to 4 ), during which artificial cerebrospinal fluid was microinjected into the cerebellum bilaterally (see Drug microinjections). These animals received three days of rest, followed by six more daily conditioning sessions (sessions 5 to 10) without any microinjection. Finally, these guinea pigs received one last session (session 11) in which muscimol was microinjected into the cerebellum bilaterally to test the effects of bilateral cerebellar inactivations on retention of the established left CRs.

A homemade computer-controlled system was used for eyeblink conditioning measurements. During drug microinjections and conditioning, guinea pigs were restrained in a cylindrical Plexiglas container $(10 \mathrm{~cm}$ in diameter and $25 \mathrm{~cm}$ in length) located in a sound- and light-attenuating chamber and their heads were fixed with blunt earbars pressing on the headstages. A speaker was placed $50 \mathrm{~cm}$ above the animal and delivered a $500 \mathrm{~ms}$ binaural tone $\mathrm{CS}(2 \mathrm{kHz}, 85 \mathrm{~dB}$ SPL, $5 \mathrm{~ms}$ rise/fall time), while a plastic pipe was placed $5 \mathrm{~mm}$ from the animal's left eyeball and delivered a $100 \mathrm{~ms}$ corneal airpuff US ( $3.0 \mathrm{psi}$, measured at the end of pipe). The onset of the airpuff US was triggered by the onset of the tone CS with a $400 \mathrm{~ms}$ interval, that is, the CS preceded and coterminated with the US (Figure 1). The daily conditioning session consisted of 50 CS-US trials grouped in five blocks with a variable intertrial interval of 20-40 s (mean $30 \mathrm{~s}$ ).

Details on the procedures of eyeblink response detection were previously described ${ }^{[21]}$. Briefly, the loop sutured into the left upper eyelid was linked by silk thread to the swivel arm of a high-resolution spring-return potentiometer (JZ-101, XH, Beijing, China). Any movement of the left upper eyelid would cause an output signal proportional to the amplitude of the left upper eyelid movement ( $1 \mathrm{mV}$ equaled $0.25 \mathrm{~mm}$ ). Eyeblink response mechanogram and markers of the applied stimuli were digitized at a sample rate of $20 \mathrm{kHz}$ in each channel by a data acquisition system (Powerlab/4sp, ADInstuments, Australia) and were acquired using the software Chart (version 5.4.2).

\section{Drug microinjections}

The $\mathrm{GABA}_{\mathrm{A}}$ receptor agonist muscimol (Sigma-Aldrich, St Louis, MO, USA) was dissolved in the sterilized aCSF prior to use. The aCSF contained (in mmol/L) $126 \mathrm{NaCl}$, $5 \mathrm{KCl}, 1.25 \mathrm{NaH}_{2} \mathrm{PO}_{4}, 2 \mathrm{MgSO}_{4}, 26 \mathrm{NaHCO}_{3}, 2 \mathrm{CaCl}_{2}$, and 10 glucose ( $\mathrm{pH} 7.3 \pm 0.1$ ). We used a dose of $1.25 \mu \mathrm{g}$ muscimol (dissolved in $1.0 \mu \mathrm{L}$ aCSF) to temporarily inactivate the unilateral cerebellum based on findings from our preliminary studies.

Drug microinjection treatments were given as shown in Table 1. Drug microinjections were made with the help of a Hamilton syringe connected by a calibrated plastic tube to a stainless steel needle inserted into the guiding cannula and were carried out at a rate of $0.2 \mu \mathrm{L} / \mathrm{min}$. The drug microinjections were performed twice at an interval of 15 min during a conditioning session. The first microinjection was made into the left side of the cerebellum, whereas the second microinjection was switched to the right side of the cerebellum. The needle tip was kept in place for $5 \mathrm{~min}$ after each microinjection to allow the drug to diffuse from the needle tip. In total, either $1.25 \mu \mathrm{g}$ muscimol or $1.0 \mu \mathrm{L}$ aCSF was microinjected into the unilateral cerebellum. The unilateral EBCC began $40 \mathrm{~min}$ after the completion of the second

Table 1. Arrangement of microinjection treatments prior to conditioning.

\begin{tabular}{|c|c|c|c|c|c|c|}
\hline & \multicolumn{2}{|c|}{ Control } & \multicolumn{2}{|c|}{ L-MSC } & \multicolumn{2}{|c|}{ R-MSC } \\
\hline & Left & Right & Left & Right & Left & Right \\
\hline $\begin{array}{l}\text { Session } 1 \\
\text { Session 2-4 }\end{array}$ & $\begin{array}{c}\text { No injection } \\
\text { aCSF }\end{array}$ & $\begin{array}{c}\text { No injection } \\
\text { aCSF }\end{array}$ & $\begin{array}{c}\text { No injection } \\
\text { muscimol }\end{array}$ & $\begin{array}{c}\text { No injection } \\
\text { aCSF }\end{array}$ & $\begin{array}{c}\text { No injection } \\
\text { aCSF }\end{array}$ & $\begin{array}{c}\text { No injection } \\
\text { muscimol }\end{array}$ \\
\hline $\begin{array}{l}\text { Session 5-10 } \\
\text { Session } 11\end{array}$ & $\begin{array}{c}\text { No injection } \\
\text { muscimol }\end{array}$ & $\begin{array}{c}\text { No injection } \\
\text { muscimol }\end{array}$ & $\begin{array}{c}\text { No injection } \\
\text { muscimol }\end{array}$ & $\begin{array}{c}\text { No injection } \\
\text { aCSF }\end{array}$ & $\begin{array}{c}\text { No injection } \\
\text { aCSF }\end{array}$ & $\begin{array}{c}\text { No injection } \\
\text { muscimol }\end{array}$ \\
\hline
\end{tabular}

Left: left side of the cerebellum; Right: right side of the cerebellum. 
microinjection.

\section{Single unit recording}

Two days after the acquisition tests, extracellular single unit recordings were performed within the left intermediate cerebellum of the R-MSC guinea pigs on session 12. This step was designed to exclude the possibility that muscimol microinjected into the right intermediate cerebellum diffused to the critical areas of the left cerebellum during the previous inactivation sessions.

Details on the procedures of single unit recording in waking guinea pigs were described previously ${ }^{[22]}$. In brief, a metal electrode (2-4 $\mu \mathrm{m}$ in diameter in tip, 2-6 M $\Omega$ impedance at $100 \mathrm{~Hz}, \mathrm{FHC}$, Brunswick, ME, USA) was automatically advanced into the left intermediate cerebellum through the left guiding cannula by a microdriver (PE-2, Narishige, Tokyo, Japan). For CR-related neuronal activity monitoring, the electrode was attached to the left guiding cannula for long-term recording. In the current study, the CR-related neuronal activities were recorded for at least $1 \mathrm{~h}$ in all R-MSC guinea pigs. For each R-MSC guinea pig, the first period (before microinjection) of single unit recording was carried out during 50 CS-US paired trials. Afterward, microinjection of muscimol into the right intermediate cerebellum was conducted. Forty minutes after completion of the right microinjection, the second period of single unit recording was performed during another $50 \mathrm{CS}$-US paired trials.

Neuronal analog signals were amplified $(\times 20$ 000) and filtered (100 and $5000 \mathrm{~Hz}$ ). Signals of neuronal activity, eyeblink response mechanogram and markers of the applied stimuli were digitized with the data acquisition system (Powerlab/4sp, ADInstuments, Australia), which sampled at $20 \mathrm{kHz}$ in each channel.

\section{Histological identification}

After the completion of all behavioral experiments, guinea pigs were deeply anesthetized $(50 \mathrm{mg} / \mathrm{kg}$ sodium pentobarbital, ip) and perfused transcardially with saline and 4\% paraformaldehyde (PFA). The cerebellum was removed and fixed in fresh PFA solution for $24 \mathrm{~h}$. After fixation, cerebellar specimens were embedded in paraffin and serial sections (10 $\mu \mathrm{m}$ thickness) were cut and mounted on adenosine phosphosulfate-coated slides. These cerebellar sections were blow-dried, deparaffinized, rehydrated and stained with toluidine blue. The locations of the guiding cannulae in the cerebellum were checked with a microscope (SMZ1500, Nikon, Japan). Only data from guinea pigs with correct guiding cannulae implantations were included in further analysis.

\section{Data analysis}

Homemade software written in the Matlab (version 6.5) environment was used to analyze the eyeblink response parameters off-line. The CR and UR were measured as the maximum extension of the left upper eyelid during a period of $200 \mathrm{~ms}$ immediately before or after the onset of the US, respectively (Figure 1). Figure 1 was an example of the raw

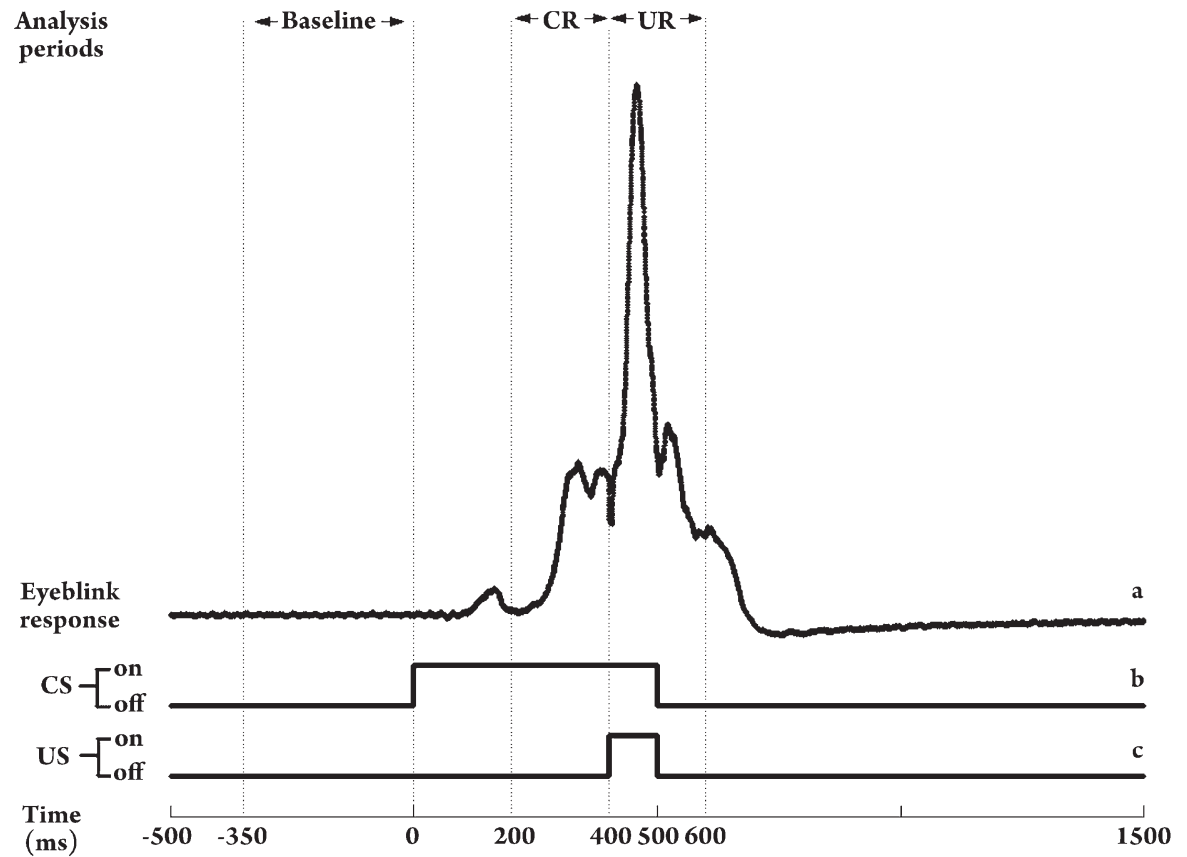

Figure 1. Temporal relationship of conditioned stimulus (CS; trace b), unconditioned stimulus (US; trace c), and analysis periods during the unilateral classical eyeblink conditioning (EBCC) in guinea pigs. In a CS-US paired trial, we analyzed the parameters of conditioned response (CR; 200-ms period before the US onset) and unconditioned response (UR; 200-ms period after the US onset). These responses were based on the average amplitude of baseline activity (350-ms period before the CS onset). An example of a typical CR and UR from a guinea pig is shown (trace a). 
left upper eyelid movement during a CS-US trial. Any upper eyelid movement exceeding the $1 \mathrm{mV}$ threshold during these periods was counted as a CR or an UR. The four eyeblink response parameters examined included (1) incidence of left CR, (2) peak amplitude of left CR, (3) onset latency of left $\mathrm{CR}$, and (4) peak amplitude of UR.

Computer-identified individual spikes were counted and summed into consecutive $10 \mathrm{~ms}$ bins for each trial. Peristimulus time histograms (PSTH) of summed unit activity for 50 CS-US paired trials (before or after the right muscimol microinjection) were conducted over a $2.0 \mathrm{~s}$ epoch, commencing $0.5 \mathrm{~s}$ prior to and $1.5 \mathrm{~s}$ following the CS onset. Means and standard errors of the firing rates in the one pretone and four post-tone $200 \mathrm{~ms}$ intervals were calculated and used to quantitatively analyze the changes in neuronal activity.

CR and UR parameters were averaged over all valid animals in each group for each training session. All data were expressed as the mean \pm SEM and were analyzed by mixed analysis of variance with Group or Time as the between subjects factor and Session or Interval as the repeated measure. In this study, one-way ANOVA with repeated measures and unpaired Student's $t$ test were also used where needed. Post hoc Newman-Keuls tests were used to analyze significant main effects or interactions when appropriate. A minimum $P$-value of $<0.05$ was used as an index of statistical significance for all tests.

\section{Results}

\section{Location of drug microinjection sites}

The locations of drug microinjection sites were checked before behavioral analysis. A guinea pig was excluded from the behavioral analysis if either tip of the two guiding cannulae was far away from the ipsilateral DCN. Figure 2A showed a representative cerebellar coronal section from a guinea pig deemed valid for the behavioral analysis. In total, 26 of the 30 guinea pigs met our inclusion criteria and their behavioral data were analyzed. Figure 2B showed the histological reconstruction of the microinjection sites in the Control $(n=8)$, L-MSC $(n=9)$ and R-MSC ( $n=9)$ groups, indicating that they were consistently close to the dorsal portion of the DCN.

\section{Left $\mathbf{C R}$ acquisition in the Control group}

In the first series of our behavioral experiments, the acquisition of left CRs in the animals without cerebellar inactivations was examined. As shown in Figure 3, the control animals $(n=8)$ demonstrated a sharp increase in the
CR incidence during the first four sessions $\left[F_{(3,21)}=70.562\right.$, $P<0.001]$. In contrast, their CR incidences during the subsequent six sessions did not differ from each other and attained an asymptotic level $\left[F_{(5,35)}=0.997, P=0.445\right]$. These results were similar to the data Kotani et al reported in intact guinea pigs $^{[23]}$. Additionally, the control animals exhibited a steady increase in the CR peak amplitude but a decrease in the CR onset latency across 10 training sessions [Peak amplitude: $F_{(9,63)}=15.979, P<0.001$; Onset latency: $F_{(9,63)}=11.431$, $P<0.001]$. On session 11, bilateral microinjections of muscimol into the cerebellum totally abolished the established left CRs in the Control group (Figures 3 and 4).

It should be noted that there was no significant difference in the CR acquisition [Incidence: $F_{(2,23)}=0.068, P=0.934$; Peak amplitude: $F_{(2,23)}=0.366, P=0.697$, Onset latency: $F$ $(2,23)=0.003, P=0.997$; see Figure 3] among the three experimental groups (Control, L-MSC and R-MSC) on session 1. This result was expected because very few CRs were displayed by either group during the initial stage of behavioral training. However, these tests were necessary for this study because varying $\mathrm{CR}$ acquisition among the three groups would weaken the validity of our subsequent findings.

\section{Left CR acquisition in the L-MSC group}

Next, the acquisition of left CRs in the animals with left cerebellar inactivation was compared with that of the control animals. As illustrated in Figure 3, the L-MSC animals $(n=9)$ showed no appreciable numbers of left CRs during sessions 2 to 4 , implying that microinjections of muscimol into the left cerebellum completely prevented expression of the left CRs. To test whether any learning had occurred during these inactivation sessions, training was continued for six more sessions without inactivation. On session 5, the second session without inactivation, the CR incidence, CR peak amplitude and CR onset latency of the L-MSC guinea pigs were indistinguishable from those of the control animals on session 2 [Incidence: $t_{(15)}=1.275, P=0.222$; Peak amplitude: $t_{(15)}=0.378, P=0.710$; Onset latency: $\left.t_{(15)}=0.949, P=0.358\right]$, indicating that no learning had occurred during the previous inactivation sessions in the L-MSC group. Therefore, in this study, sessions 5 to 10 after left cerebellar inactivation in the L-MSC group were termed the 'equivalent normal session 2 to 7 .

We then compared the CR acquisition of the L-MSC animals during the 'equivalent normal session 2 to 7 ' with that of the control animals during sessions 2 to 7 . A mixed ANOVA with the factors Group and Session revealed no significant effect of Group [Incidence: $F_{(1,15)}=0.682, P=0.422$; Peak amplitude: $F_{(1,15)}=0.360, P=0.558$; Onset latency: $F_{(1,15)}$ 
$\mathbf{A}$

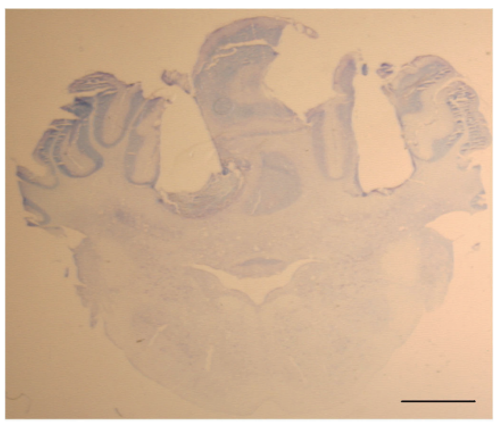

B
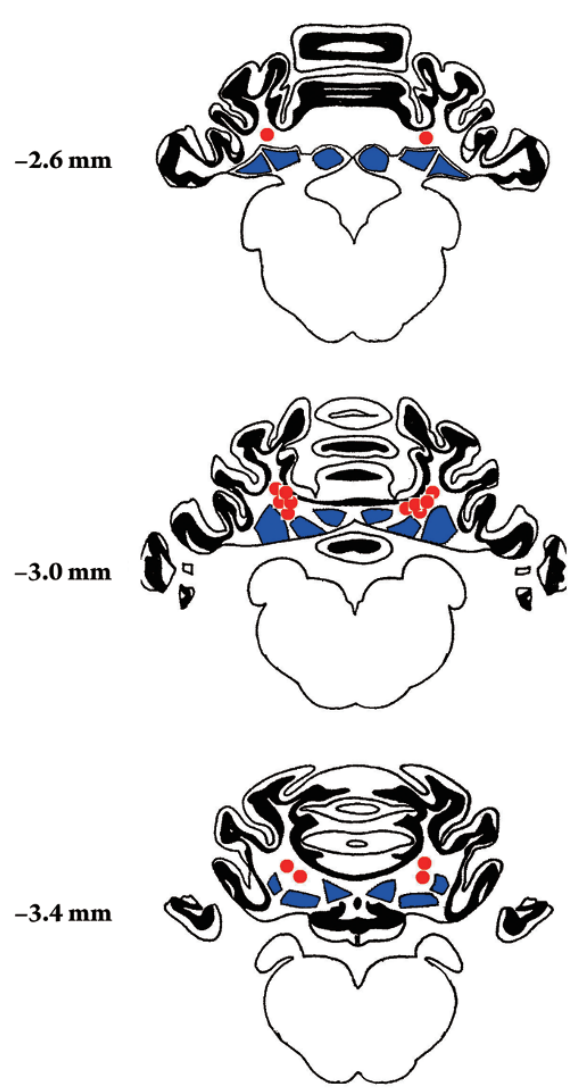

L-MSC
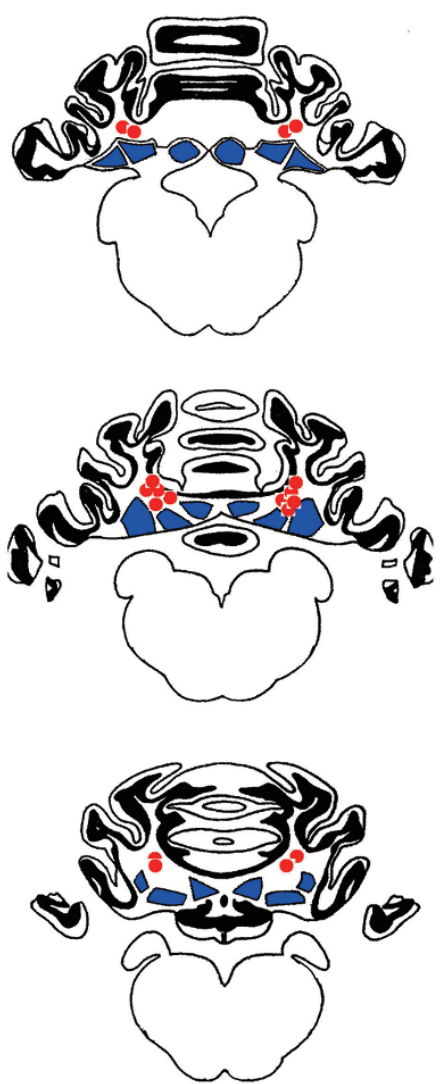

R-MSC
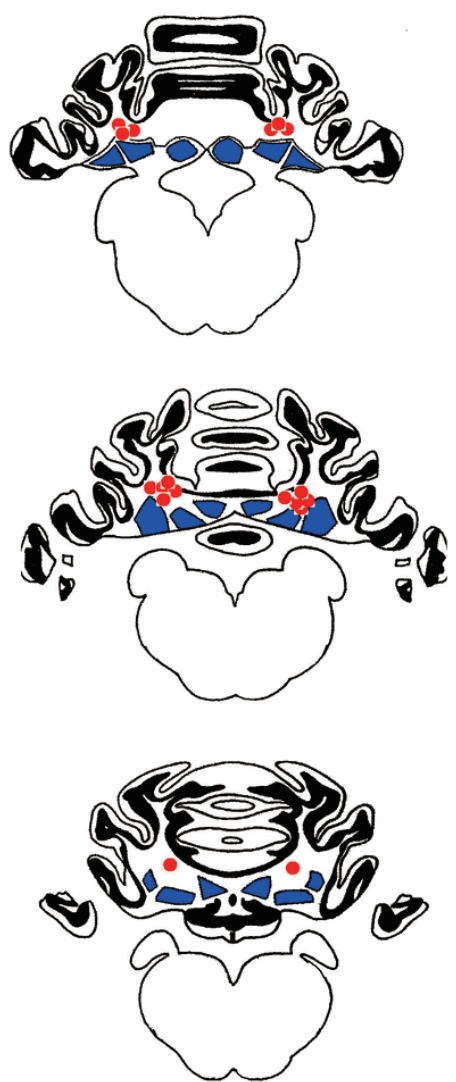

Figure 2. Location of the drug microinjection sites in the cerebellum of guinea pigs. (A) Representative toluidine blue-stained coronal cerebellar section $(10 \mu \mathrm{m})$ from a guinea pig that received drug microinjections. Two guiding cannulae pass through the ipsilateral and contralateral cerebellar cortex and their tips lay immediately dorsal to the deep cerebellar nuclei (DCN). Scale bar represents $2.0 \mathrm{~mm}$. (B) Line drawings of coronal sections showing the location of the drug microinjection sites (red circles) in the Control ( $n=8)$, L-MSC ( $n=9)$ and R-MSC ( $n=9)$ groups, respectively. Black areas represent the cerebellar cortex, whereas blue areas represent the DCN. Numbers represent the distance (mm) between the sections and frontal zero plane.

$=1.158, P=0.229$ ] or interaction [Incidence: $F$ value $=1.263$, $P=0.297$; Peak amplitude: $F$ value $=0.288, P=0.801$; Onset latency: $F$ value $=1.653, P=0.198]$, but in this case a strong effect of Session was found [Incidence: $F$ value $=47.035, P$ $<0.001$; Peak amplitude: $F$ value $=37.758, P<0.001$; Onset latency: $F$ value $=11.047, P<0.001]$. These results indicated that (1) significant acquisition of the left CRs occurred during these training sessions in either group and (2) during the 'equivalent normal session 2 to 7', the L-MSC animals exhibited the same incidence, peak amplitude, and onset latency of left CR as those of the control animals during sessions 2 to 7. On session 11, microinjections of muscimol into the left 
cerebellum completely abolished the established the left CRs in the L-MSC group (Figures 3 and 4).

\section{Left CR acquisition in the R-MSC group}

Finally, the acquisition of left CRs in the animals with right cerebellar inactivations was compared with that of the control animals. As shown in Figures 3 and 4, the R-MSC animals $(n=9)$ acquired the left CRs during sessions 2 to 4 $\left[F_{(2,16)}=61.516, P<0.001\right]$ and reached an incidence level of $96.7 \% \pm 1.9 \%$ on session 5 . The data from sessions 2 to 4 were then analyzed. A mixed-design ANOVA with factors Group and Session revealed a strong effect of Session [Incidence: $F_{(2,30)}=78.562, P<0.001$; Peak amplitude: $F_{(2,30)}=18.353$, $P<0.001$; Onset latency: $\left.F_{(2,30)}=34.845, P<0.001\right]$. This result indicated that animals in both groups acquired the left CRs during sessions 2 to 4 . A significant main effect for Group was also noted [Incidence: $F_{(1,15)}=16.735, P=0.001$; Peak amplitude: $F_{(1,15)}=7.843, P=0.013$; Onset latency: $\left.F_{(2,30)}=9.486, P=0.008\right]$. Follow-up post hoc analysis with the Newman-Keuls test further revealed that the control animals outperformed the R-MSC guinea pigs on session 2 $\left[P_{s}<0.05\right.$ for incidence, peak amplitude and onset latency of left $C R]$, but not during sessions 3 and 4, indicating a retardation of the left CR acquisition at the early stage of training in the R-MSC group. Moreover, a significant Session $\mathrm{X}$ Group interaction effect was found for the $\mathrm{CR}$ incidence $\left[F_{(2,30)}=9.100, P=0.001\right]$ but not for the CR peak amplitude $\left[F_{(2,30)}=0.171, P=0.324\right]$ and $C R$ onset latency $\left[F_{(2,30)}=2.613\right.$, $P=0.090]$. On session 11 , microinjections of muscimol into the right cerebellum had no effect on the performance of the established left CRs in the R-MSC group (Figures 3 and 4).

\section{Effects of drug microinjections on the performance of UR}

In this study, the effects of drug microinjections on the performance of tone-airpuff evoked UR were also examined. Our statistical results revealed that there were no significant differences among the UR peak amplitudes of the control animals before, during and after bilateral aCSF microinjections $\left(F_{(2,14)}=0.607, P=0.559\right.$, Figure 5$)$, suggesting that bilateral microinjections of aCSF into the cerebellum did not affect the performance of tone-airpuff evoked UR. Similarly, microinjections of muscimol into either the left or the right cerebellum did not affect the performance of tone-airpuff evoked UR. This conclusion was confirmed by our statistical results, which revealed that there were no significant differences among the UR peak amplitudes of the L-MSC animals before, during and after the left cerebellar inactivations $\left[F_{(2,16)}=2.262, P=0.136\right.$, Figure 5]. Also, there were no differ-
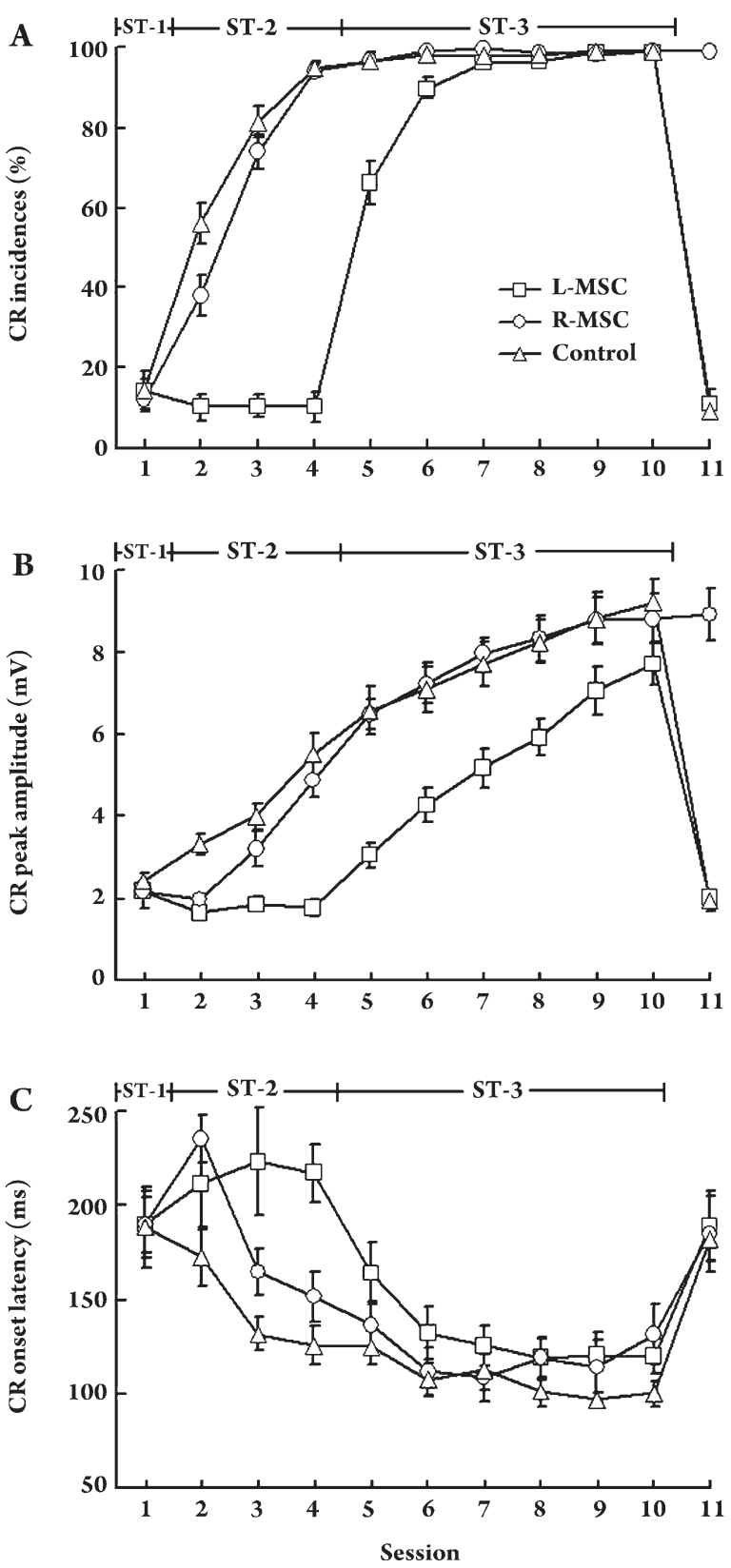

Figure 3. Effect of drug microinjection on the acquisition and performance of left CRs. (A) CR incidence, (B) CR peak amplitude and (C) CR onset latency recorded from the Control (circles), L-MSC (squares), and R-MSC (triangles) groups, respectively, across eleven conditioning sessions. ST-1 (stage 1) includes session 1 , whereas ST-2 (stage 2) includes sessions 2 to 4 , during which the drug microinjections were performed. ST-3 (stage 3 ) includes sessions 5 to 10 , during which the conditioning was carried out without any drug microinjection. Symbols are the same for all three charts. Data are given as mean \pm SEM.

ences among the UR peak amplitudes of the R-MSC animals before, during and after the right cerebellar inactivations 

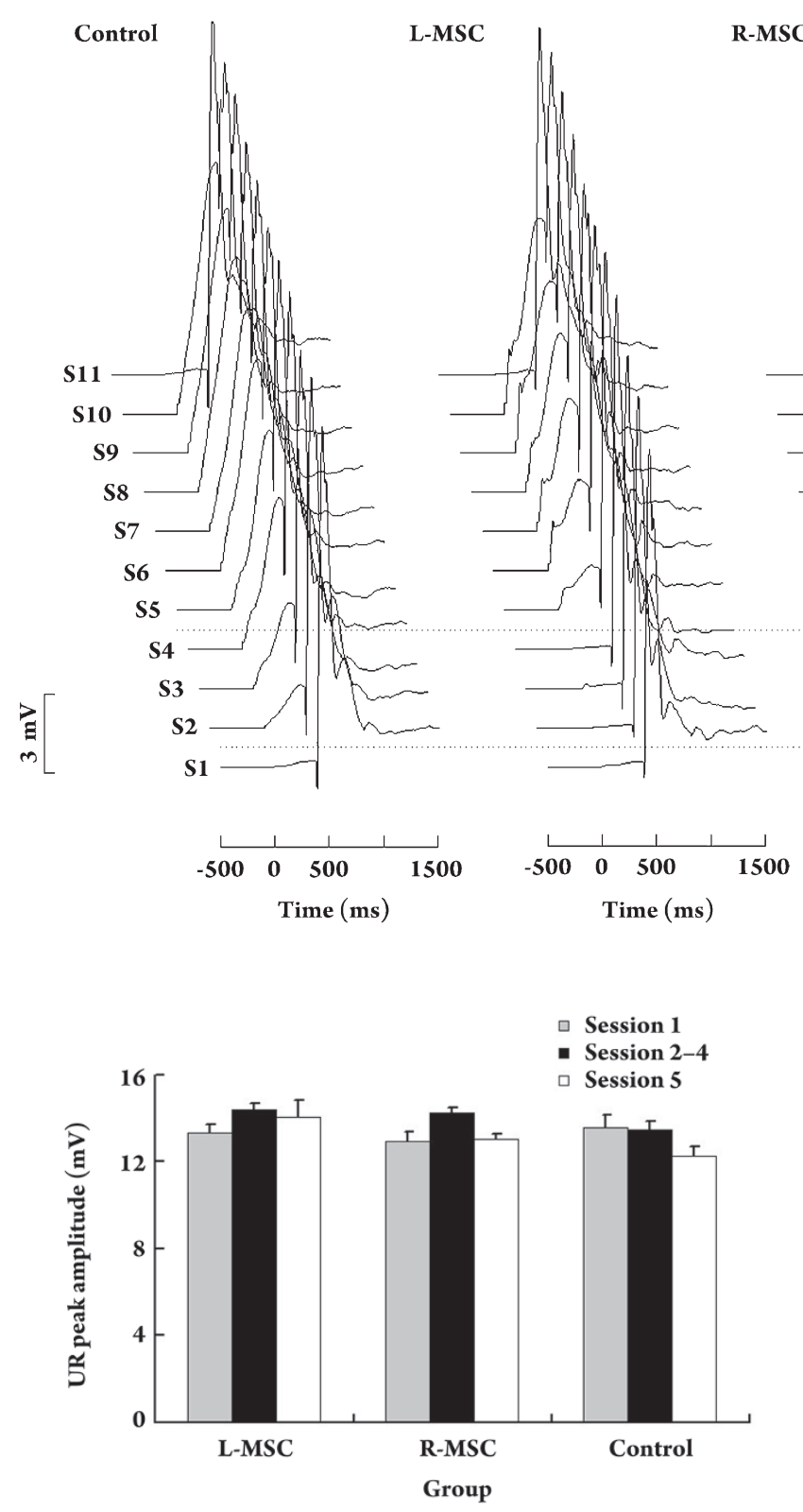

Figure 5. Effect of drug microinjection on the performance of UR. UR peak amplitudes measured for three experimental groups before (session 1; gray solid bar), during (sessions 2 to 4 ; black solid bar), and after (session 5; open bar) drug microinjections were illustrated. Data are given as mean \pm SEM.

$\left[F_{(2,16)}=0.141, P=0.870\right.$, Figure 5]. Because there was no evident correlation between the degree of CR impairment and change of UR performance across the L-MSC and R-MSC groups, the suggestion that inactivation impaired CRs by affecting performance, rather than due to loss or retardation of learning, is not supported.
Figure 4. Averaged responses of CSUS trials of the Control (left), L-MSC (middle) and R-MSC (right) groups across eleven conditioning sessions (S1-S11). Each stack plot represents a complete printout of eyeblinks from $\mathrm{S} 1$ to $\mathrm{S} 11$. The CS started at $0 \mathrm{~ms}$, whereas the US started at $400 \mathrm{~ms}$. The bidirectional arrow represents the sessions with drug microinjections.

\section{Effect of right MSC microinjection on left neuro- behavioral activity}

We next wanted to ensure that the retardation of left CR acquisition in the R-MSC group on session 2 simply reflected the effect of right cerebellar inactivation instead of a false positive effect of partial left cerebellar inactivation. We compared the left neuro-behavioral activity before and after the right muscimol microinjection on session 12 to determine whether muscimol microinjected into the right intermediate cerebellum diffused to the critical areas of the left intermediate cerebellum during the previous inactivation sessions.

Figure $6 \mathrm{~A}$ illustrated the peri-stimulus time histograms (PSTHs, trace $\mathrm{b}$ ) of a representative CR-related neuron in the left intermediate cerebellum before and after the right muscimol microinjection. In total, nine valid neurons were pooled together for analysis of mean firing rates before and after right muscimol microinjection (see Figure 6C). A mixed ANOVA with factors Time (before or after right muscimol microinjections) and Interval (five 200-ms interval) revealed no significant effect of Time $\left[F_{(1,16)}=0.002, P=0.965\right]$ or interaction $[F$ value $=0.217, P=0.868]$, but in this case a strong effect of Interval was found $[F$ value $=121.962, P<0.001]$. This result suggested that microinjection of a dose of $1.25 \mu \mathrm{g}$ muscimol into the right intermediate cerebellum did not affect the neuronal activity in the left intermediate cerebellum. Moreover, Figure $6 \mathrm{~B}$ showed that the right muscimol microinjection 


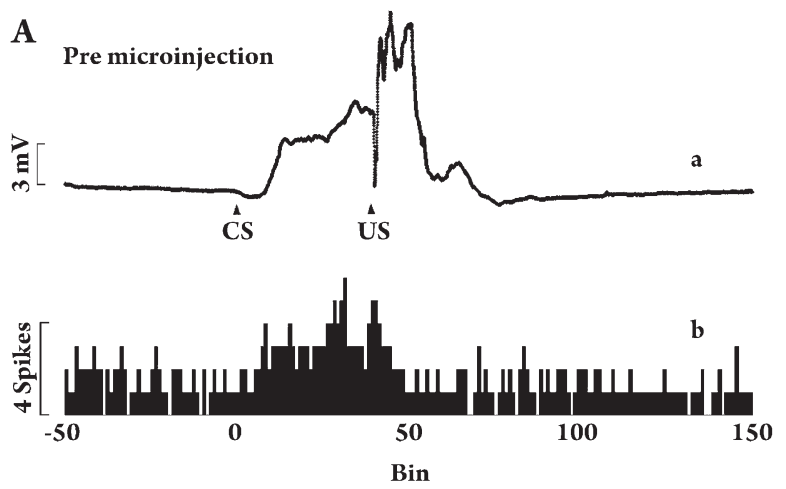

B
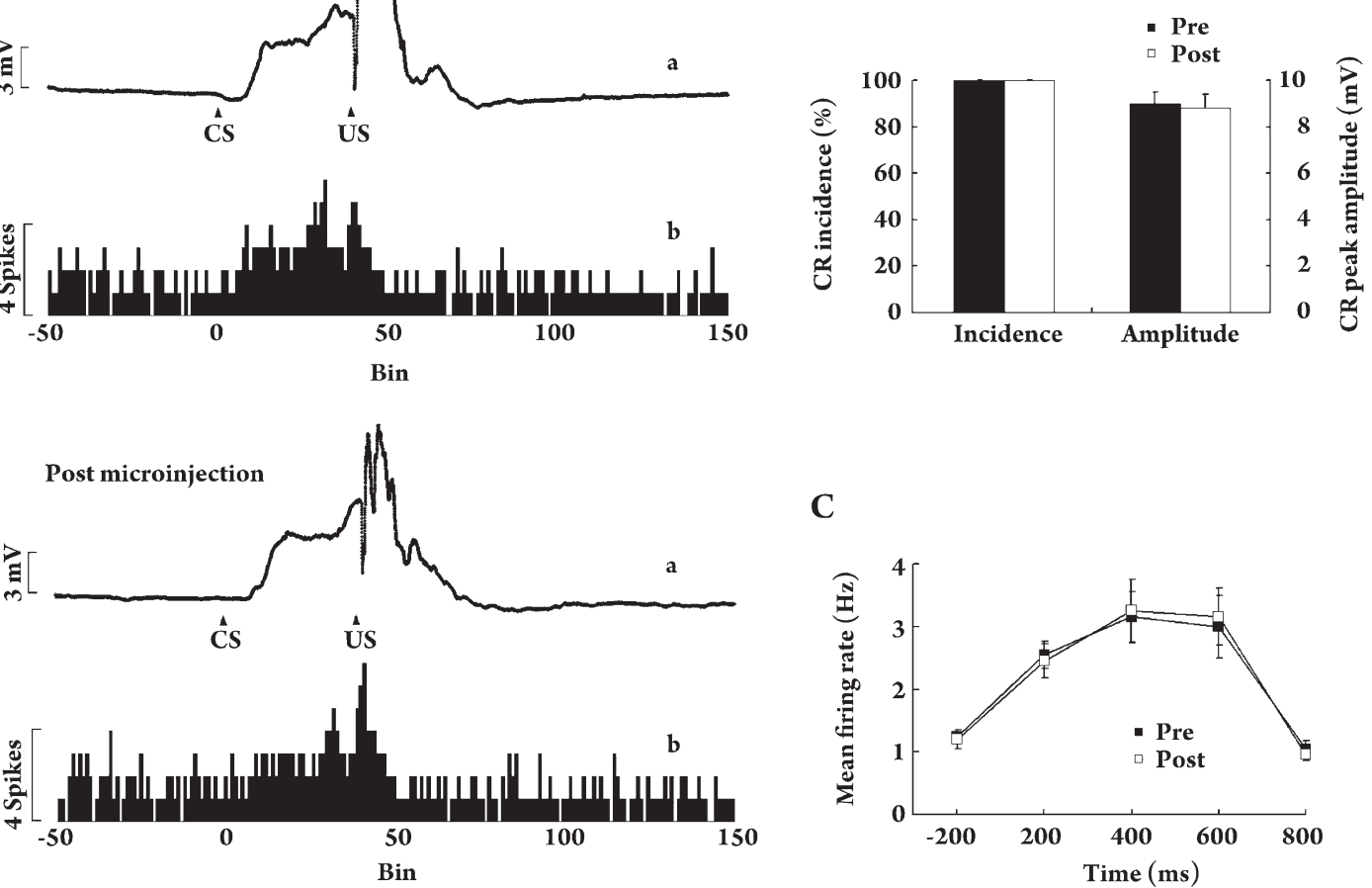

Figure 6. Effect of right muscimol microinjection on left neuro-behavioral activity. (A) Peri-stimulus time histograms (PSTH) recorded from a neuron of left intermediate cerebellum in a valid R-MSC guinea pig before (upper; trace b) and after (bottom; trace b) right muscimol microinjection. Bin width for these histograms was $10 \mathrm{~ms}$. Averaged responses of CS-US trials of the same animal before (upper; trace a) and after (bottom; trace a) the right muscimol microinjection were also illustrated. (B) CR performance before (solid bars) and after (open bars) the right muscimol microinjection. (C) Mean firing rates of the left DCN neurons before (solid circles) and after (open circles) the right muscimol microinjection. Data are given as mean \pm SEM.

did not change the performance of established left CRs [Incidence: $t_{(8)}=0.000, P=1.000$; peak amplitude: $t_{(8)}=0.312$, $P=0.763]$. Based on the neuro-behavioral data, it seemed unlikely that muscimol microinjected into the right intermediate cerebellum diffused to the critical areas of the left intermediate cerebellum in R-MSC animals during the previous inactivation sessions.

\section{Discussion}

The primary findings of the current study can be summarized as follows: (1) microinjections of muscimol, a $\mathrm{GABA}_{\mathrm{A}}$ receptor agonist, into the left cerebellum before training completely prevented acquisition of the left CRs in albino guinea pigs. (2) Microinjections of muscimol into the right cerebellum before training retarded acquisition of the left CRs at the early stage of training, whereas CR acquisition was not affected at the later stage of training relative to that of the control animals. (3) Microinjections of either aCSF or muscimol into the cerebellum did not affect the performance of tone-airpuff evoked UR.

\section{Extent of muscimol inactivation in the unilateral cer-}

\section{ebellum}

To test the unilateral cerebellar involvement in the acquisition of unilateral EBCC, reversible inactivations of the critical areas of the unilateral cerebellum had to be complete. In the current study, we used a dose of $1.25 \mu \mathrm{g}$ musicmol to temporarily inactivate the critical areas of the unilateral cerebellum. A similar or even smaller dose of muscimol was previously reported to have a complete inactivation effect ${ }^{[8,9,24]}$. In addition, the well-established left CRs of the L-MSC animals were totally abolished by the inactivation of the left cerebellum on session 11. Consequently, we believe that our dose of muscimol is fully sufficient to inactivate the unilateral cerebellum.

A microinjection volume of $1.0 \mu \mathrm{L}$ muscimol occupies a sphere of radius $\sim 3.0 \mathrm{~mm}^{[25]}$. Therefore, in this study, 
the spread of muscimol in the unilateral cerebellum should be estimated. Krupa et al ${ }^{[8,24]}$, using ${ }^{3} \mathrm{H}$-labeled muscimol autoradiography, reported that the maximal radial spread of a dose of $1.40 \mu \mathrm{g}$ muscimol in the unilateral cerebellum was $\sim 3.0 \mathrm{~mm}$, similar to the muscimol spread reported by Martin et al in the cerebellar cortex ${ }^{[26]}$. Assuming a similar uptake and distance for the spread of muscimol from the microinjection sites located in the close vicinity of the DCN, some areas of the cerebellar cortex might be affected in the current study. According to the results suggested by some previous studies, cerebellar cortical function is unlikely to be normal even if the muscimol microinjections were completely restricted to the $\mathrm{DCN}^{[9,20]}$. Loss of nucleo-olivary inhibition and disruption of nucleo-cortical inputs would disturb the function in the olivo-cortico-nuclear compartment ${ }^{[27,28]}$. For these reasons, in the current study we made no particular attempt to restrict the spread of muscimol to the vicinity of the DCN and our procedures should be viewed as a general inactivation of the unilateral cerebellum.

\section{Role of the ipsilateral cerebellum in the acquisition of unilateral EBCC}

The cerebellum has been shown to be critical for the acquisition of $\mathrm{CR}$ in rabbits ${ }^{[8,29]}$ and mice $\mathrm{e}^{[12,30,31]}$. In the current study, learning of CS-US association was completely prevented during the paired conditioning with inactivations of the left cerebellum, as evidenced by the same left CR acquisition level of the L-MSC animals on session 5 relative to that of the control animals on session 2. Furthermore, the L-MSC and the control animals acquired the left CRs with very similar rates during the subsequent equivalent normal sessions. These results confirmed the findings in both rabbits and mice, indicating that the essential role of the ipsilateral cerebellum during the acquisition of unilateral EBCC was conserved among various species.

\section{Role of the contralateral cerebellum in the acquisi- tion of unilateral EBCC}

To fully understand the possible contralateral cerebellar involvement in the acquisition of unilateral EBCC, two questions should be addressed: The first one is whether there are anatomical pathways via which contralateral cerebellar plasticity can be generated. It was previously reported that a pathway existed via which the unilateral US signals could be relayed to both sides of the cerebellum ${ }^{[32,33]}$, implying that the signals of unilateral US could converge with the signals of bilateral CS on both sides of the cerebellum. According to the learning theories of Albus and Marr ${ }^{[34,35]}$, it seemed reasonable that plasticity could be generated in the unilateral cerebellum contralateral to the stimulated side of the US during the unilateral EBCC. Up to now, there some data have indirectly suggested the existence of this contralateral plasticity ${ }^{[10-15]}$. Moreover, there are anatomical pathways through which the unilateral cerebellum could control bilateral eyeblink responses. Although the contralateral eyeblink responses were smaller in size and longer in latency, stimulation of the brachium conjunctivum of the unilateral cerebellum could activate bilateral orbicularis oculi muscles ${ }^{[36]}$.

The other question is whether the contralateral cerebellar plasticity is substantially involved in acquisition of the ipsilateral CRs. Because some data indirectly suggest a contralateral regulation of acquisition or expression of the ipsilateral CRs, we decided to further assess the contralateral cerebellar involvement. In the current study, the R-MSC animals acquired the left CRs by session 4, whereas their learning was significantly retarded on session 2 . On session 12 , microinjections of muscimol into the right cerebellum had no effect on left neuro-behavioral activity in the R-MSC group. Together with the findings in the L-MSC group, our results indicate not only that the left $\mathrm{CR}$ acquisition was mainly dependent on the left cerebellum, but also that the right cerebellum was potentially involved in the acquisition of left CRs during the early stage of training.

Strikingly, the CR acquisition of R-MSC animals was similar to that of control animals starting at session 3. Furthermore, inactivation of the right cerebellum on session 11 failed to change the incidence and peak amplitude of the established left CRs of the R-MSC guinea pigs, indicating that the right cerebellum was not essential for either the acquisition of left CRs at the later stage of training or the performance of well-established left CRs. These results implied a changing involvement of the right cerebellum in the left CR acquisition across behavioral training in albino guinea pigs, which fits well with evidence obtained from functional imaging studies in rabbits ${ }^{[11]}$ and molecular biological studies in mice ${ }^{[12]}$. In the fMRI study of rabbits, significant learningrelated increases of the blood oxygenation level-dependent (BOLD) response were observed early in training within the bilateral cerebellar cortex and DCN. However, later in training, the significant learning-related BOLD response remained bilateral within the cerebellar cortex and predominant in the ipsilateral DCN. Accumulating evidence has indicated that the cerebellar cortex is involved in modulating some aspects (eg, timing ${ }^{[37,38]}$ or rate ${ }^{[39,40]}$ ) of the CR, whereas the DCN are the essential locus supporting the acquisition of $\mathrm{CR}^{[8,24]}$. Based on previous findings, we inferred that the changing involvement of contralateral DCN in the acquisition of unilateral EBCC was one of the possible mechanisms underly- 
ing the changing contralateral involvement.

\section{Conclusion}

Combined with findings from previous studies, results from the current study support the assertions that (1) the contralateral cerebellum is potentially involved in the acquisition of unilateral EBCC, especially at the early stage of training, and (2) plasticity occurring within the ipsilateral cerebellum, rather than within the contralateral cerebellum, is essential for the acquisition of unilateral EBCC.

\section{Acknowledgements}

This project was supported by grants from the National Natural Science Foundation of China (No 30672255 and 30771769).

We thank Prof Hai-wei XU and Prof Ying XIONG from the Department of Physiology, Third Military Medical University, for their detailed comments and suggested improvements to the manuscript. We also thank Prof Ming-kui LUO from the Department of Math, Third Military Medical University, for his helpful assistance with statistical analysis.

\section{Author contribution}

Jian-feng SUI and Hua FENG designed the research; Bo HU, Xi LIN, and Lü-shuai HUANG performed the research; Bo HU and Li YANG analyzed data; Bo HU and Li YANG wrote the paper.

\section{References}

1 Christian KM, Thompson RF. Neural substrates of eyeblink conditioning: acquisition and retention. Learn Mem 2003; 11 : 427-55.

2 Rogers RF, Britton GB, Steinmetz JE. Learning-related interpositus activity is conserved across species as studied during eyeblink conditioning in the rat. Brain Res 2001; 905: 171-7.

3 Green JT, Arenos JD. Hippocampal and cerebellar single-unit activity during delay and trace eyeblink conditioning in the rat. Neurobiol Learn Mem 2007; 87: 269-84.

4 Lincoln JS, McCormick DA, Thompson RF. Ipsilateral cerebellar lesions prevent learning of the classically conditioned nictitating membrane/eyelid response. Brain Res 1982; 323: 190-3.

5 Lavond DG, Hembree TL, Thompson RF. Effect of kainic acid lesions of the cerebellar interpositus nucleus on eyelid conditioning in the rabbit. Brain Res 1985; 326: 179-82.

6 Welsh JP, Harvey JA. Pavlovian conditioning in the rabbit during inactivation of the interpositus. J Physiol (London) 1991; 444: 459-80

7 Clark RE, Zhang AA, Lavond DG. Reversible lesions of the cerebellar interpositus nucleus during acquisition and retention of a classically conditioned behavior. Behav Neurosci 1992; 106: 879-88.

8 Krupa DJ, Thompson JK, Thompson RF. Localization of a memory trace in the mammalian brain. Science 1993; 260: 98991.

9 Hardiman MJ, Ramnani N, Yeo CH. Reversible inactivations of cerebellum with muscimol prevent the acquisition and extinction of conditioned nictitating membrane responses in the rabbit. Exp Brain Res 1996; 110: 235-47.

10 McCormick DA, Lavond DG, Clark GA, Kettner RE, Rising CE, Thompson RF. The engram found? Role of the cerebellum in classical conditioning of nictitating membrane and eyelid responses. Bull Psychon Soc 1981; 18: 103-5.

11 Miller MJ, Chen NK, Li BL, Tom B, Weiss C, Disterhoft JF, et al. fMRI of the conscious rabbit during unilateral classical eyeblink conditioning reveals bilateral cerebellar activation. J Neurosci 2003; 23: 11753-8.

12 Park J, Onodera T, Nishimura S, Thompson RF, Itohara S. Molecular evidence for two-stage learning and partial laterality in eyeblink conditioning of mice. Proc Natl Acad Sci USA 2007; 103: 5549-54.

13 Logan CG, Grafton ST. Functional anatomy of human eyeblink conditioning determined with regional cerebellar glucose metabolism and positron-emission tomography. Proc Natl Acad Sci USA 1995; 92: 7500-4.

14 Blaxton TA, Zeffiro TA, Gabrieli JD, Bookheimer SY, Carrillo $\mathrm{MC}$, Theodore $\mathrm{WH}$, et al. Functional mapping of human learning: a positron-emission tomography activation study of eyeblink conditioning. J Neurosci 1996; 16: 4032-40.

15 Dimitrova A, Weber J, Maschke M, Elles HG, Kolb FP, Forsting $\mathrm{M}$, et al. Eyeblink-related areas in human cerebellum as shown by fMRI. Hum Brain Mapp 2002; 17: 100-15.

16 Krogsgaard-Larsen P, Johnston GA. Structure-activity studies on the inhibition of GABA binding to rat brain membrane by muscimiol and related compounds. J Neurochem 1978; 30: 13771382.

17 Palacios JM, Young WS, Kuhar MJ. Autoradiographic localization of $\gamma$-aminobutyric acid (GABA) receptors in the rat cerebellum. Proc Natl Acad Sci USA 1980; 77: 670-4.

18 Sastry BR, Morishita W, Yip S, Shew T. GABA-ergic transmission in deep cerebellar nuclei. Prog Neurobiol 1997; 53: 259-71.

19 Rapisarda C, Bacchelli B. The brain of the guinea pig in stereotaxic coordinates. Arch Sci Biol 1977; 61: 1-37.

20 Yeo $\mathrm{CH}$, Lobo DH, Baum A. Acquisition of a new-latency conditioned nictitating membrane response- major, but not complete, dependence on the ipsilateral cerebellum. Learn Mem 1997; 3: 557-77.

$21 \mathrm{Hu}$ B, Lin X, Xiong Y, Huang W, Liu G, Sui J. Activity of single granule cell in the dentate gyrus of guinea pig during the consolidation of trace eyeblink conditioned responses. Prog Biochem Biophys 2006; 33: 1051-60.

$22 \mathrm{Hu}$ B, Yang L, Huang W, Sui J. A comparison of the spontaneous firing patterns between principal cells and fast spiking interneurons from dentate gyrus in waking guinea pigs. Neurosci Bull 2006; 22: 21-8.

23 Kotani S, Kawahara S, Kirino Y. Trace eyeblink conditioning in decerebrate guinea pigs. Eur J Neurosci 2003; 17: 1445-54. 
24 Krupa DJ, Thompson RF. Reversible inactivation of the cerebellar interpositus nucleus completely prevents acquisition of the classcially conditioned eye-blink response. Learn Mem 1997; 3: 545-56.

25 Edeline JM, Hars B, Hennevin E, Cotillon N. Muscimol diffusion after intracerebral microinjections: a reevaluation based on electrophysiological and autoradiographic quantifications. Neurobiol Learn Mem 2002; 78:100-24.

26 Martin J. Autoradiographic estimation of the extent of reversible inactivation produced by microinjection of lidocaine and muscimol in the rat. Neurosci Lett 1991; 24: 160-4.

27 Katz DB, Steinmetz JE. Sigle-unit evidence for eye-blink conditioning in cerebellar cortex is altered, but no eliminated, by interpositus nucleus lesions. Learn Mem 1997; 3: 88-104.

28 Bengtsson F, Hesslow G. Cerebellar control of the inferior olive. Cerebellum 2006; 5: 7-14.

29 McCormick DA, Thompson RF. Cerebellum: essential involvement in the classically conditioned eyelid response. Science 1984; 223: 296-9.

30 Bao S, Chen L, Kim JJ, Thompson RF. Cerebellar cortical inhibition and classical eyeblink conditioning. Proc Natl Acad Sci USA 2002; 99: 1592-7.

31 Wada N, Kishimoto Y, Watanabe D, Kano M, Hirano T, Funabiki $\mathrm{K}$. Conditioned eyeblink learning is formed and stored without cerebellar granule cell transmission. Proc Natl Acad Sci USA 2007; 104: 16690-5.
32 Morcuende S, Delgado-Garcia JM, Ugolini G. Neuronal premotor networks involved in eyelid responses: retrograde transneuronal tracing with rabies virus from the orbicularis oculi muscle in the rat. J Neurosci 2002; 22: 8808-18.

33 Van Ham JJ, Yeo CH. Somatosensory trigeminal projections to the inferior olive, cerebellum and other precerebellar nuclei in rabbits. Eur J Neurosci 1992; 4: 302-17.

34 Albus JS. A theory of cerebellar function. Math Biosci 1971; 10: 25-61.

35 Marr D. Theory of cerebellar cortex. J Physiol 1969; 202: 437-70.

36 Ivarsson M, Hesslow G. Bilateral control of the orbicularis oculi muscle by one cerebellar hemisphere in the ferret. Neuroreport 1993; 4: 1127-30.

37 Bao S, Chen L, Kim JJ, Thompson RF. Cerebellar cortical inhibition and classical eyeblink conditioning. Proc Natl Acad Sci USA 2002; 99: 1592-7.

38 Garcia KS, Mauk MD. Pharmacological analysis of cerebellar contribution to the timing and expression of conditioned eyelid responses. Neuropharmacology 1998; 37: 471-80.

39 Lavond DG, Steinmetz JE. Acquisition of classical eyeblink conditioning without cerebellar cortex. Behav Brain Res1989; 33: 113-64.

40 Attwell PJE, Rahman S, Yeo CH. Acquisition of eyeblink conditioning is critical dependent on normal function in cerebellar cortical lobule HVI. J Neurosci 2001; 21: 5715-22. 